\title{
Dilatation of benign anastomotic strictures after colorectal surgery with bougies
}

\author{
Mürşit Dincer
}

Department of General Surgery, Istanbul Haseki Training and Research Hospital, Istanbul, Turkey

Submitted: 5 March 2018

Accepted: 13 September 2018

Arch Med Sci Civil Dis 2018; 3: e121-e123

DOI: https://doi.org/10.5114/amscd.2018.81058

Copyright @ 2018 Termedia \& Banach

\section{Abstract}

Introduction: The development of benign anastomotic strictures after colon-rectum operations is one of the complications. Endoscopic balloon and bougie dilatations are applied primarily in the treatment. In this study, we aimed to investigate the causes of benign anastomosis after low anterior resection and present the results of bougie dilatation in anastomosis stenosis. Material and methods: The patients with anastomosis after low anterior resection in the last year were retrospectively evaluated. Anastomosis and dilatation after the development of the cases were examined. The patients who underwent bougie dilatation were included in the study. Demographic characteristics of the patients, indications of surgery, causes of stenosis, duration of development of stenosis, level of stenosis and number of operations were recorded. Dilatation was performed under sedation. Polyvinyl bougies with a guide wire were used during the procedure. The dilatation process was continued starting with the smallest diameter bougie that could pass through the anastomosis, up to the maximum diameter bougie. Results: After low anterior resection, seven patients with anastomosis were included in the study. In four cases, the only successful session was after the bougie dilatation. In one case, anastomosis was detected after multiple operations. In two cases, the bougie dilatation failed.

Conclusions: As a result, bougie dilatation in anastomotic stenosis is a feasible, effective and safe minimally invasive method with a low complication rate.

Key words: colonoscopy, postoperative complications, anastomosis, strictures, dilatation.

\section{Introduction}

The development of benign anastomotic strictures after colon-rectum operations is one of the complications. It is a frequent complication especially after low anterior resection. Incidence varies between $5 \%$ and $10 \%$. This ratio is up to $20 \%$ in anastomoses made with staples [1-4]. It is usually seen after anastomosis leakages. Such factors as radiotherapy, the fact that anastomosis is made with staples, bleeding in the anastomosis line, inflammation and leakage are the causes of the aetiology of benign stenosis [5]. Endoscopic balloon and bougie dilatations are applied primarily in the treatment. In this study, we aimed to investigate the causes of benign anastomosis after low anterior resection and present the results of bougie dilatation in anastomosis stenosis.

\author{
Corresponding author: \\ Mürşit Dincer \\ Department \\ of General Surgery \\ Istanbul Haseki \\ Training and \\ Research Hospital \\ 34100 Istanbul, Turkey \\ Phone: +905446422820 \\ E-mail: drmursitdincer@ \\ gmail.com
}




\section{Material and methods}

The patients with anastomosis after low anterior resection in the last year were retrospectively evaluated. Anastomosis and dilatation after the development of the cases were examined. The patients with anastomosis stenosis and balloon dilatation at control endoscopies were excluded from the study. The patients who underwent bougie dilatation were included in the study. Demographic characteristics of the patients, indications of surgery, causes of stenosis, duration of development of stenosis, level of stenosis and number of operations were recorded. Dilatation was performed under sedation. Polyvinyl bougies with a guide wire were used during the procedure. The dilatation process was continued starting with the smallest diameter bougie that could pass through the anastomosis, up to the maximum diameter bougie. Each bougie was inserted up to the proximal part of the anastomosis with the aid of a guide wire and left for $2 \mathrm{~min}$. The procedures were performed blindly without fluoroscopy. The opening of the passage after the operation and the endoscope's easily being inserted into the proximal part of the anastomosis were evaluated as success. All patients were checked for a possible complication with physical examination after the operation. The success rates, number of procedures and complications were recorded.

\section{Results}

After low anterior resection, seven patients with anastomosis were included in the study. Five cases were men and two cases were women. The mean age was 55.2 (range: 37-84). Four cases of rectal adenocarcinoma, one case of rectal polyps, and two cases of rectal invasion due to endometriosis were operated on. Anastomosis developed at an average of 4.1 months (range: 2-12 months). Anastomosis leakage in three cases, radiotherapy in two cases, and the use of staples in two cases were noted in aetiology. In four cases, the only successful session was after the bougie dilatation. In one case, anastomosis was detected after multiple operations. In two cases, the bougie dilatation failed. In 2 patients, anastomosis leakage was observed after low anterior resection due to rectal cancer. In one case, although the opening of the passage with bougies was achieved, the symptoms did not improve, and again the permanent stoma was opened. In the other case, bougie dilatation was insufficient to expand the granulation tissue and the narrowing could not be opened.

\section{Discussion}

Benign anastomotic stenosis is a condition that occurs with symptoms such as gas-stool failure and distension in the abdomen after a contraction in the lumen which does not allow the passage of the endoscope after colon-rectum surgery $[6,7]$. It has an incidence of $5-10 \%$ after low anterior resection. It is usually seen in the early postoperative period [8]. In the aetiology, such factors as anastomotic leakages, radiotherapy, the presence of an ischaemic anastomosis, and anastomotic staples are specified $[9,10]$. There are publications that indicate increased incidence of stenosis after use of a stapler [4, 6]. In our study, anastomosis leakage in three cases developing anastomosis stenosis, radiotherapy in two cases, and staples use in two cases were noted in the aetiology. Most of the narrowness is restored by itself and closing of the deflector stoma and passing of stool in the column segment ensure that the passage remains open. Despite all this, benign anastomosis can produce unwanted results. In some cases, deflector stoma can become permanent stoma. Treatment is especially difficult in anastomotic stenosis developing due to anastomosis leakage and radiotherapy. In the treatment of anastomosis stenosis, while surgical resection was in the foreground in the past years, endoscopic dilatations are applied primarily today. Stent applications may allow the opening of the passage in cases where the treatment is not possible with endoscopy [8]. The last treatment option is surgery in cases where there is no solution with minimal invasive approaches. However, the success rate of relaparotomy is also low in these cases.

There are three types of endoscopic dilator. These are balloon dilators, bougies filled with mercury or tungsten, and guide wire polyvinyl bougies [11]. Dilatation can be performed under fluoroscopy or blindly. In blindly treated procedures, the performance rates are higher than the other procedures. This ratio is $0.3-0.4 \%$. Since fluoroscopy cannot be performed in our Endoscopy Unit, we have been performing dilatation operations blindly. However, no perforation has ever been encountered to date.

Araujo et al. reported the success rate in the disease series as $97 \%$ after balloon dilatation [12]. Again, Placer et al., in a study of 26 patients developing anastomosis after anterior resection, reported the balloon dilatation success rate as $88.5 \%$ [7]. In a study comparing successfully treated patients with anastomosis with endoscopy applied once, there are publications that indicate that balloon dilatation is more effective than bougie dilatation [13]. Balloon dilatation reduces the risk of perforation most because the pressure is evenly distributed. Despite all this, our balloon dilatation success rates in benign anastomosis stenosis, which developed after low anterior resection, were lower in our previous experience. For this reason, we are now applying bougie dilatation 
in such stenoses. In a study of 15 patients with benign anastomosis after low anterior resection by Werre et al., the success rate of bougie dilatation was reported as $67 \%$ [2]. In our study, this rate was $71.4 \%$.

Araujo et al. reported a rate of re-dilatation of only $18.2 \%$ after 2 years of follow-up in patients undergoing dilation in their study [12]. In our study, more than one session of dilatation was performed in three patients at the end of 1 year follow-up (42.8\%). In one of these cases, a successful result was obtained, and in two cases the operations failed. In 2 patients for whom we failed, anastomosis leakage was observed after low anterior resection due to rectal cancer. In one case, although the opening of the passage was achieved with a bougie, the symptoms did not improve, and again the permanent stoma was opened. In the other case, bougie dilatation was insufficient in expending the granulation tissue and the narrowness could not be opened. Such complications as bleeding and perforation can occur after the bougie dilatation. The risk of perforation is reported to be $0-3.8 \%$ in the literature $[6,7,12,14]$. In our study, cases of minor bleeding not requiring replacement were seen. However, no perforation developed in any of the cases. The negative side of this study is the number of cases. However, the number of centres experienced in the management of these cases after anastomosis is not high. In the literature, there are no publications in which there are very wide series.

As a result, bougie dilatation in anastomotic stenosis is a feasible, effective and safe minimally invasive method with a low complication rate. In addition, to minimize anastomosis leakage to be sensitive, to target a stress-free anastomosis, to try to close the deflector stomas early, is easier than the efforts to correct anastomosis narrowness. A war against anastomosis stenosis can be avoided by a smaller fight to prevent the development of stenosis.

\section{Conflict of interest}

The author declares no conflict of interest.

\section{References}

1. Luchtefeld MA, Milsom JW, Senagore A, Surrel JA, Mazier WP. Colorectal anastomotic stenosis: results of a survey of the ASCRS membership. Dis Colon Rectum 1989; 32: 733-6.

2. Werre A, Mulder C, Van Heteren C, Spillenaar B. Dilation of benign strictures following low anterior resection using Savary-Gilliard bougies. Endoscopy 2000; 32: 385-8.

3. Geller A, Gal E. Dilation of benign strictures following low anterior resection using Savary-Gilliard bougies. Endoscopic treatment of benign anastomotic colorectal stenosis with electrocautery. Gastrointest Endosc 2001; 54: 277-9.
4. Marchena Gomez J, Ruiz de la Cuesta E, Gomez Guerra G, Vallejo Gallego I, Garcia-Anguiano F, Hernandez Romero JM. Anastomotic stricture with the EEA-Stapler after colorectal anastomosis. Rev Esp Enferm Dig 1997; 89: 835-42.

5. Hirono S, Ueno M, Takifuji K, et al. Successful interventional dilatation of a complicated stricture of the anastomotic colon: report of a case. Intern Surg 2007; 92: 311-3.

6. Di Giorgio P, De Luca L, Rivellini G, Sorrentino E, D'Amore E, De Luca B. Endoscopic dilation of benign colorectal anastomotic stricture after low anterior resection: a prospective comparison study of two balloon types. Gastrointest Endosc 2004; 60: 347-50.

7. Placer C, Urdapilleta G, Markinez I, et al. Benign anastomotic strictures after oncologic rectal cancer surgery. Results of treatment with hydrostatic dilation. Cir Esp 2010; 87: 239-43.

8. Delaunay K, Barthelemy C, Dumas O, Balique JG, Audigier JC. Endoscopic therapy of benign colonic postoperative strictures: report on 27 cases. Gastroenterol Clin Biol 2003; 27: 610-3.

9. Chung RS, Hitch DC, Armstrong DN. The role of tissue ischemia in the pathogenesis of anastomotic stricture. Surgery 1988; 104: 824-9.

10. Ragg J, Garimella V, Cast J, Hunter IA, Hartley JE. Balloon dilatation of benign rectal anastomotic strictures - a review. Dig Surg 2012; 29: 287-91.

11. Ginzburg L, Greenwald D, Cohen J. Complications of endoscopy. Gastrointest Endosc Clin N Am 2007; 17: 405-32.

12. Araujo SE, Costa AF. Efficacy and safety of endoscopic balloon dilation of benign anastomotic strictures after oncologic anterior rectal resection: report on 24 cases. Surg Laparosc Endosc Percutan Tech 2008; 18: 565-8.

13. Pietropaolo V, Masoni L, Ferrara M, Montori A. Endoscopic dilation of colonic postoperative strictures. Surg Endosc 1990; 4: 26-30.

14. Ambrosetti P, Francis K, Peyer R, Frossard JL. Colorectal anastomotic stenosis after elective laparoscopic sigmoidectomy for diverticular disease: a prospective evaluation of 68 patients. Dis Colon Rectum 2008; 51 : 1345-49. 